\title{
ANN Based Admission Control for On-Chip Networks
}

\author{
Boqian Wang \\ KTH Royal Institute of Technology \\ National University of Defense Technology \\ boqian@kth.se
}

\author{
Zhonghai Lu \\ KTH Royal Institute of Technology \\ zhonghai@kth.se
}

\author{
Shenggang Chen \\ National University of Defense \\ Technology \\ shec@kth.se
}

\begin{abstract}
We propose an admission control method in Network-on-Chip (NoC) with a centralized Artificial Neural Network (ANN) admission controller, which can improve system performance by predicting the most appropriate injection rate of each node via the network performance information. In the online control process, a data preprocessing unit is applied to simplify the ANN architecture and make the prediction results more accurate. Based on the preprocessed information, the ANN predictor determines the control strategy and broadcasts it to each node where the admission control will be applied. Compared to the previous work, our method builds up a high-fidelity model between the network status and the injection rate regulation. The full-system simulation results show that our proposed method can enhance application performance by $17.8 \%$ on average and up to $23.8 \%$.
\end{abstract}

\section{INTRODUCTION}

With the development of on-chip technologies, more cores and larger NoC are provided, which means abundant resources to support parallel applications. But it also means a more complex circumstance to reasonably manage resources, increasing utilization and avoiding collision. It is the traffic diversity and unpredictability that raise complexity and performance uncertainty in orders of magnitude [22]. To address this problem, traffic regulation was proposed where traffic flows or streams can be shaped into desired characteristics before being admitted into the interconnect [14][10][15]. Then, the leaky bucket model can be used to preferably shape traffic using a mathematical formalism which restricts the injection rate $\rho$ of each node. In this way, it can help to mitigate traffic congestion as well as reallocate hardware resources, improving both network and application performance.

Although flow regulation via admission control is shown to be an effective method in improving $\mathrm{NoC}$ and system performance, building up a mathematical relationship between the NoC status and an appropriate admission control scheme is hard. The current NoC status has many aspects such as injection rates, throughput, latency, global and partial congestion status, etc, making the relationship a very complex one to be established. Also, we need to consider future circumstances, requesting a predictive algorithm to make prediction. Besides, the diversity of application characteristics almost makes it impossible to propose explicit equations which can capture this relationship. In contrast, the ANN model offers an alternative tool, which can build up an implicit relation among complex inputs and

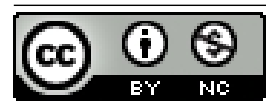

This work is licensed under a Creative Commons Attribution-NonCommercial International 4.0 License.

DAC '19, fune 2-6, 2019, Las Vegas, NV, USA

(C) 2019 Copyright held by the owner/author(s).

ACM ISBN 978-1-4503-6725-7/19/06

https://doi.org/10.1145/3316781.3317772 outputs. Also, ANN can act as a predictor, which is suitable for a diversity of cases, fulfilling all the requests listed above.

In this paper, we propose a centralized ANN Admission Controller (ANN-AC). The Network Interface (NI) of each node is responsible for periodically collecting network status information in a certain sampling window (epoch) and sending it to the ANN-AC. In the ANN-AC, a data preprocessing unit is applied for grouping nodes and offering integrated information to the ANN predictor. This preprocessing unit can greatly reduce architecture complexity of the ANN predictor model so that it can achieve higher prediction accuracy with less training data. Besides, a control rate generator is applied after the ANN predictor, producing admission control information based on the ANN output and broadcasting it to each node. In summary, our work has the following contributions:

- We design an ANN based admission control system which can enhance application performance by building up the current network status and the most appropriate admission control strategy for each node.

- We propose a preprocessing unit and a feature point extraction method to customize ANN model inputs so that it can achieve higher prediction accuracy with less training data.

- We show the feasibility of our proposed admission controller via a low-overhead hardware implementation and analyze its area and power consumption by hardware synthesis.

The rest of the paper is organized as follows. Related works are discussed in Section 2. In Section 3, we present background on NoC admission control. Section 4 details the design of our proposal and Section 5 discusses its implementation. After reporting experimental results in Section 6, we conclude in Section 7.

\section{RELATED WORK}

Flow regulation (traffic shaping) was earlier proposed to deal with the NoC QoS problem [14][10]. The flow regulation method can be categorized into a dynamic or static one, depending on whether the parameters, burstiness $(\sigma)$ and rate $(\rho)$, are determined dynamically or statically. While the conventional static methods [14][10][18][1] are cheaper to implement, their rigid principles can only be applied to limited scenarios and often result in deviated performance. Therefore, the dynamic approaches are proposed to enhance the prediction accuracy and control applicability. Kaxiras et al. [13] and Huang et al. [8] both propose a table-driven approach to make prediction on one future time interval and achieve higher performance. Thomas et al. [20] and Lu et al. [15] proposed a fuzzy logic based algorithm which is applied on NoC to adapt the traffic pattern according to the historic status information. In [15], a predictor is embedded in each NI which is located between the client core and the network. The predictor projects the values of $\rho$ and $\sigma$ for the next time window based on the information collected by a sliding window mechanism. Then, a fuzzy controller is proposed, using prediction results to dynamically control packet injection from each client node to the network [15]. This flow regulation mechanism 
can be used on an open-loop or a closed-loop scenario which both can lead to performance improvement.

Different from the conventional methods, ANN as well as other learning methods is proposed to achieve the desired predictive control [16]. It has been used in branch prediction [19], hotspots prediction in NoCs [12], CMP power management [23][11], memory controller's self-optimizing [9], resources allocation [5] and power-aware NoC design with learning enabled sleepy storage links and routers [6]. Bitirgen et al. [5] proposed an ANN based predictor to allocate the NoC resources. The authors formulate global resource allocation as a machine learning problem, which manages multiple shared CMP resources to enforce higher-level performance objectives by learning each application's performance response to different resource distributions.

\section{BACKGROUND ON ADMISSION CONTROL}

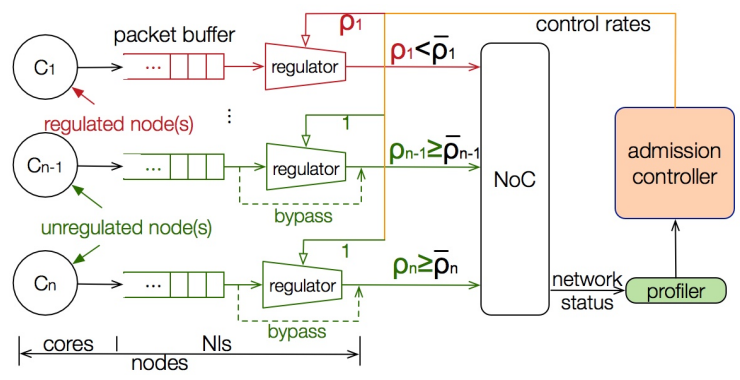

Figure 1: A centralized admission control process

In the admission control process, as shown in Figure 1, cores inject packets into buffers, where packets have to get admission from the flow regulator before being sent into NoC. The regulator sets the maximum injection rate of each node to the value of received control rate. Assuming that the original injection rate without regulation mechanism is expressed by $\bar{\rho}$ and injection rate with flow regulation mechanism is expressed by $\rho$, for the rate regulated core $C_{1}$ in this figure, its injection rate will be limited to $\rho_{1}$ which is between $(0,1)$ and smaller than $\bar{\rho}_{1}$. For the rate unregulated node $C_{n-1}$ and $C_{n}$, the control rate will be set as 1 , which indicates a bypass for packet transfer. In this case, $\rho_{n-1}$ and $\rho_{n}$ are only restricted by the NoC congestion status. Because the decrease of the injection rate for $C_{1}$ will mitigate the NoC congestion status and leave more resources to packets from $C_{n-1}$ and $C_{n}, \rho_{n-1}$ and $\rho_{n}$ can be bigger than (or at least equal to) the original $\bar{\rho}_{n-1}$ and $\bar{\rho}_{n}$ If the overall increase of the injection rate in unregulated nodes is larger than its decrease in regulated nodes, the throughput and the system performance improves. The control rates, which are produced by the admission controller basing on the status collected by the profiler, are used to increase the overall network throughput.

In the previous work [15], Lu and Yao proposed a fuzzy controller to adaptively regulate the packet flow of each node, which achieves a higher performance network. However, with the fuzzy control algorithm, it can only gradually adjust the regulation level step by step. Thus, it is slow in reacting to network congestion status. In this paper, we inventively propose an ANN based Admission Controller (ANN-AC) to determine the flow regulation rates. Compared to the previous works, it builds up a more effective model which can quickly adjust regulated rates to an ideal case according to network performance status, achieving larger performance improvement.

\section{ANN BASED ADMISSION CONTROL DESIGN} 4.1 Design overview

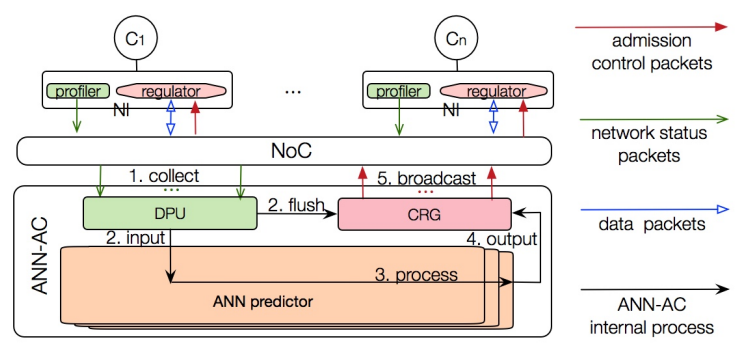

Figure 2: ANN based admission control system

Figure 2 gives an overview of the proposed ANN based admission control system. In each NI, we use a profiler to collect network status information via the packets the NI sends or receives. The regulator in each $\mathrm{NI}$ is responsible for receiving injection regulation information and applying admission control. The regulation information is decided by an ANN based admission controller (ANN-AC), which includes a data preprocessing unit (DPU), a control rate generator $(\mathrm{CRG})$ and a kernel ANN predictor. The sequence of data flow between each unit inside the ANN-AC is marked by numbers. We summarize the function of each unit below:

- DPU: It collects and preprocesses network status information sent from all nodes and then, offers the input data to ANN predictor. At the same time, it will also calculate nodes grouping information according to the injection rate of each node and flush results to CRG.

- ANN predictor: It takes input data from DPU and gives out a predictive injection regulation level to CRG.

- CRG: After receiving the predictive regulation level from the ANN predictor, it generates control rates according to the predictive level and nodes grouping information and then, broadcasts them to each node.

\subsection{Data Preprocessing Unit (DPU)}

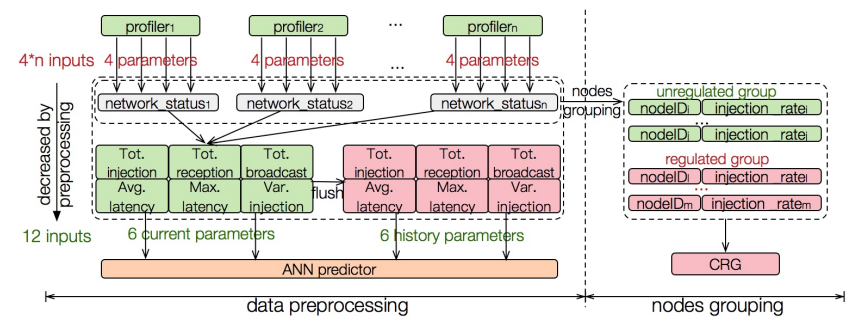

Figure 3: Data collection and preprocessing in DPU

As shown in Figure 3, DPU will receive network status information from each node, which contains four kinds of parameters: average latency of received packets, number of injected packets, number of received packets and broadcast number, comprehensively recording the network status in spatial dimension. Especially, the broadcast number is used to record the number of multi-response/request packets triggered by a single request packet, which will cause partial traffic block in the sending or receiving node. For example, if a write request from a L1 cache to the shared L2 cache triggers a broadcast invalidation request to 3 other L1 caches which have copies of the requested data, then the original requestor L1 cache will receive 3 more response packets from $\mathrm{L} 1$ caches due to cache 
coherence protocol. In this case, the broadcast number for this request is 3 .

After that, DPU will extract injection rates information from all nodes, based on which the nodes grouping decision will be made and flushed to CRG for further use. At the same time, DPU will preprocess network status information and offer the ANN predictor the most efficient information as input data which can reflect the current network status, network status development tendency as well as the adjustment space of injection rates. Nodes grouping makes it possible for collecting ANN training data and is also a method which can significantly diminish the output complexity of ANN architecture while achieving relatively high performance improvement. Data preprocessing is the method we apply to extract the most useful network status information to decrease the input nodes of ANN predictor and increase the prediction accuracy.

4.2.1 Nodes grouping. In the traditional admission control process, different nodes will receive different admission control rates. But for the ANN model training process, it is almost impossible to test all those possibilities to find the best control strategy. As an alternative, we use a grouping method, which will spit all nodes into two groups based on the injection rate, i.e., the number of injected packets per epoch. Nodes in the first group with low packet injection will be unregulated. But for nodes in the second group which has high packet injection, they will receive regulated control rates. By nodes grouping, we separate the nodes with high injection rates apart and apply a unified control strategy to them. This method only asks for a single result which can greatly simplify the training data extraction process and assure a higher prediction accuracy, bringing more benefit for performance enhancement.

As shown in Figure 4, we present an ideal example from all test results (see detailed configurations in Section 6.1) to illustrate our nodes grouping strategy. We calculate the threshold $\left(T_{h}\right)$ according to Equation 1 by the lowest $\left(\rho_{l}\right)$ and the highest $\left(\rho_{h}\right)$ packet injection rates among all nodes.

$$
T_{h}=\rho_{l}+0.7 \times\left(\rho_{h}-\rho_{l}\right)
$$

All the nodes with injection rate being lower than $T_{h}$ belong to the unregulated group and the rest the regulated group. Here, 0.7 is an empirical value obtained by trials with real application benchmarks, which can help to make decision on threshold so that, in most cases, the nodes with high packet injection will be separated into the regulated group.

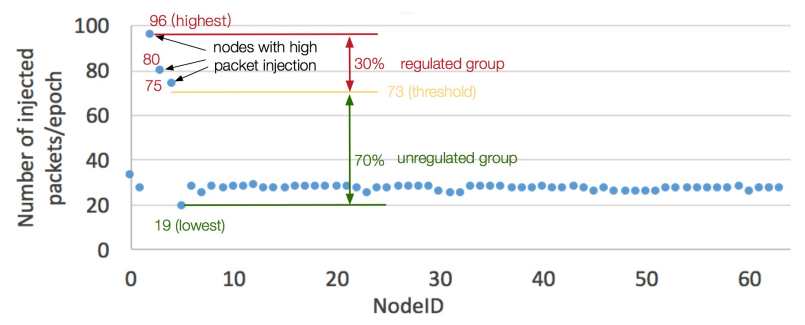

Figure 4: Nodes grouping

The grouping information which contains nodeID and injection rate of each node will be flushed into CRG, waiting for the prediction result from ANN predictor to make the final control rate decision.

4.2.2 Input Data preprocessing. In this step, the received 4 kinds of different network statues information (average latency of received packets, number of injected packets, number of received packets and broadcast number) from all nodes will be integrated into ANN as the input data. Originally, in an $8 \times 8$ mesh network, the information totally contains $256(=64 \times 4)$ data per sampling window. To make the prediction more accurate, we should decrease the ANN input parameters and extract the most significant information which should reflect the network current status (spatial dimension), the tendency of network status (time dimension) and the adjustment degree we can apply to the regulated group (result space).

Based on the original 256 data from all nodes, we utilize 6 more efficient parameters to comprehensively fulfill the requirements mentioned above, including total injected packets (Tot.injection), total received packets (Tot.reception), total broadcast number (Tot.broadcast), average packet latency (Avg.latency), maximum packet latency (Max. latency), which reflect the network status from spatial dimension and variance of average injected packets (Var.injection) between two groups, which reflect the adjustment space we can apply to nodes in the regulated group. To consider the nature of continuity in the network performance, the DPU will store the last-epoch history results for those six parameters, which could describe the network status in the time dimension. The current data and the history data together form the input data, being injected into the ANN predictor. In this way, the number of ANN input parameters is significantly reduced from 256 to 12 , while still including the most useful information for making prediction and control decision. In this way, we can achieve higher prediction accuracy with less training data. In the experimental section, we compare the prediction accuracy results between the 256 and 12 inputs ANN models.

\subsection{ANN Predictor}

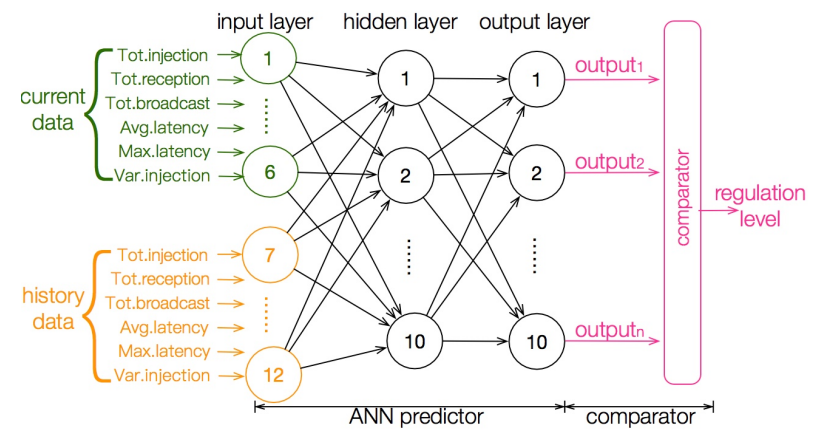

Figure 5: ANN predictor architecture

4.3.1 The ANN predictor model. We propose a multi-layer feedforward ANN [7] model to build up the ANN predictor as shown in Figure 5. The ANN predictor has twelve input nodes, six of which receive current data and the rest history data. It has ten outputs, each standing for a regulation level from 1 to 10 . The final regulation level is selected by the comparator according to Equation 2.

$$
\text { regulation_level }=\operatorname{Index}\left(\max \left(\text { output }_{1}, \ldots, \text { output }_{10}\right)\right)
$$

The rest configuration is shown in Table 1. We use the Gaussian function in each neural node to calculate the node output value and once the square error of the predictive results is within the error threshold, the training process ends. The selection of those parameters is a trade-off decision which is mainly based on the tactics that the accuracy should be assured and at the same time decreasing the offline training set, online execution latency and the consumption of area and power as much as possible. 
Table 1: The ANN predictor model

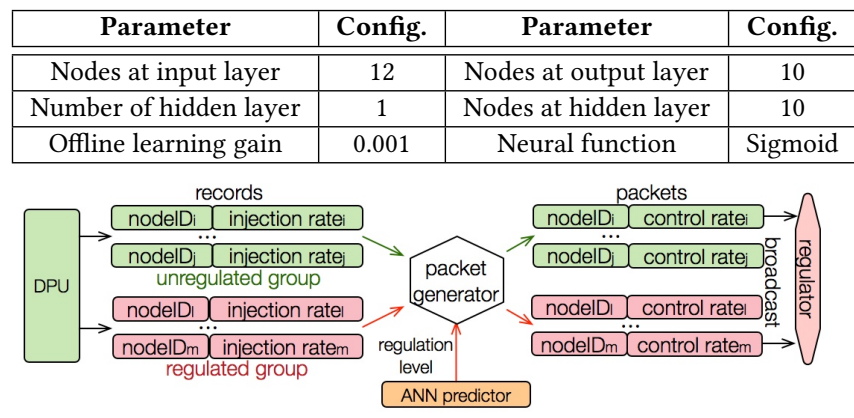

Figure 6: The CRG architecture

\subsection{Control Rate Generator (CRG)}

As shown in Figure 6, it takes node grouping information from DPU and the regulation level result from the ANN predictor as inputs. Basing one those information, the packet generator will generate admission control packets which include control rates. The control rate is calculated by Equation 3. Divided by 10, the predictive regulation level $[1,9]$ will be normalized between $[0.1$, $0.9]$, which will then be multiplied by the injection rate collected in the last sampling window to generate a new control rate for the corresponding node. One exception is that if the predictive regulation level equals to 10 , the control rate will be set to 1 , which indicates an unregulated control rate. The packets will be finally broadcast to all nodes according to the nodeID information. The distributed flow regulator in each NI will execute the admission control strategy based on the received control rate.

$$
\begin{aligned}
& \text { ctrl_rate }(T+1)=i n j \_r a t e(T) \times \frac{\text { level }}{10}, \quad \text { if level } \in[1,10) \\
& \text { ctrl_rate }(T+1)=1, \quad \text { if level }=10
\end{aligned}
$$

\section{IMPLEMENTATION OF ANN BASED ADMISSION CONTROL SYSTEM}

\subsection{ANN model training process}

We trained our ANN predictor offline with training data extracted by running the PARSEC benchmarks [3] in full-system simulator GEM5 [4] with the system configurations shown in Section 6.1. The extracted data from all benchmarks in PARSEC form the training data example. Each data sample contains 12 input data and 10 output data. The dataset generation process is shown in Figure 7.

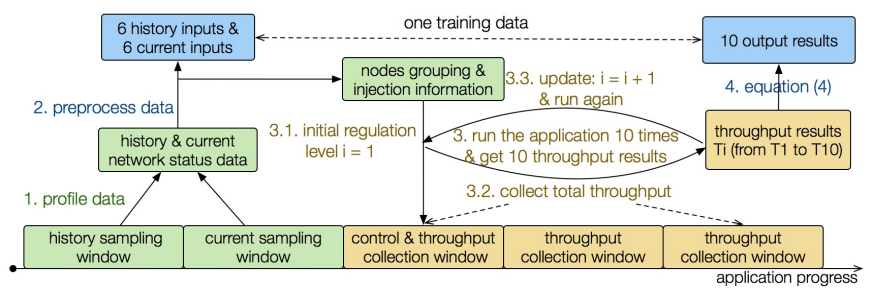

Figure 7: Training data generation process

We first get the corresponding 6 historical inputs, 6 current inputs and nodes grouping result by profiling and preprocessing data collected in history and current sampling windows. Then, by iteratively running the application 10 times with 10 different regulation levels (from 1 to 10) being applied to the nodes in regulated group, we correspondingly get 10 throughput results generated by the next three windows after the current sampling window. Finally, assuming that $T_{i}$ is the result collected when applying regulation level $i, T_{\max }$ and $T_{\min }$ are the respective maximum and minimum throughput values among the 10 results, the 10 output data is calculated by Equation 4 . This equation shows the principle that a higher throughput value indicates a higher chance the corresponding control level will be chosen. The 12 inputs and 10 outputs together form one training data sample. From the whole program execution traces, we evenly extract training data from sampling windows which are picked up according to nine feature points. These feature points capture the three time periods when the average packet injection is relatively low, middle, or high in one benchmark, and the six changing phases between the three injection levels.

$$
\text { output }_{i}=\frac{T_{i}-T_{\min }}{T_{\max }-T_{\min }}, i \in[1,10]
$$

Compared with the one-output design, the ten-output architecture combined with a selector can tolerate the fluctuation of predicted results and thus can also contribute to overfitting prevention. Besides, during the training process, we also use the early stopping method to prevent overfitting. Specifically, the strategy we used is called "No-improvement-in-n", where the best validation accuracy will be recorded during training and the iteration will be stopped when the accuracy results from continual $n$ epochs are less than the best one in record.

\subsection{Centralized implementation}

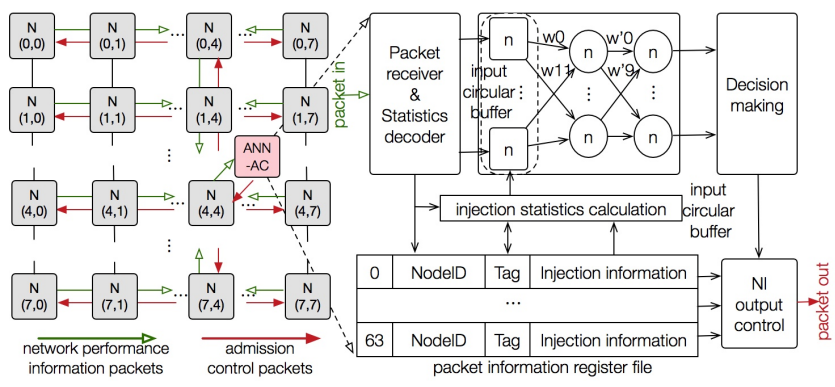

Figure 8: Implementation of ANN-AC in NoC architecture

As shown in Figure 8, the ANN-AC is attached to one of the central routers in the $8 \times 8$ mesh NoC, which decreases average communication distance and response latency. In a X-Y routing based NoC, the communications between the ANN-AC node and other nodes are represented by arrow lines. We have made an RTL implementation of ANN-AC, the system configuration of which is the same as the one we implement in GEM5. Hardware implementation of ANN-AC consists of three parts: peripheral part (including statistics decoder unit, decision making unit and output control unit) which is mainly responsible for numerical calculations, storage part (including input circular buffer and packet information register file) and ANN kernel part (ANN predictor). In the peripheral part, the numerical calculation functions such as grouping and averaging are realized by approximate solutions. The function of dividing 10 , for example, can be approximated by $(1 / 2)^{4}+(1 / 2)^{5}+(1 / 2)^{8}+(1 / 2)^{9}$ $(\approx 0.1)$ and thus implemented by the shifter and adder to decrease the hardware design complexity. In the storage part, the packet information register file is used to record the group and injection information, which includes nodeID (6 bits), injection information (10 bits) and tag (1 bit) for grouping. The depth of the register is 
64 and it overall takes 1088 bits $((6+10+1) \times 64)$. The extracted information will be stored in the input circular buffer, participating in the current and next ANN prediction process. The input circular buffer overall stores 12 inputs (each 10 bits), taking 120 bits $(12 \times 10)$. As for the ANN predictor, we adapt multi-level network, separating the convolution and nonlinear functions into different execution cycles to improve calculating speed. According to the characteristics of weight and bias results produced by training process, we use a 16-bit signed fixed point number to represent all weights and bias parameters so that their precision is $(1 / 2)^{14}$ and the final predictive results are also acceptable. Besides, we use the combination logic based approximation method [21] to simulate the hardware realization of the nonlinear sigmoid function. The detailed hardware synthesis results are presented in Section 6 .

\subsection{Implementation feasibility}

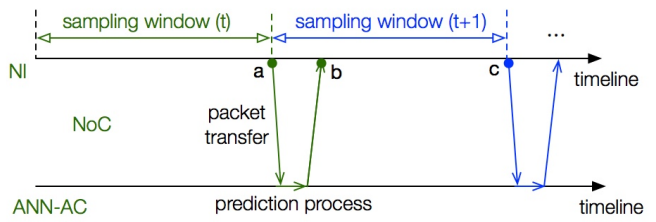

Figure 9: The interaction between the NI and the ANN-AC

By re-using the existing NoC for communications between the ANN-AC and cores (without introducing a new NoC or a bus), the centralized implementation incurs low overhead. Yet the communication overhead and ANN-AC response time have to be considered for feasibility. To this end, Figure 9 shows the interaction timeline between NI and the ANN-AC. Once a sampling window ends, a node will send out information packets to the ANN-AC node at time $a$ and receive the control packets at time $b$. Since the control rate is used for the whole sampling window (from time $a$ to time $c)$, assuming that the injection rate during time $a$ and $b$ is $I R_{a b}$, then the admission rate for the rest period $I R_{b c}$ is determined by Equation 5. In this way, the communication and calculation latency caused by centralized control mechanism can be overlapped, contributing to the enhancement of system scalability and releasing the requirement of ANN-AC latency. In this context, the calculation latency of our designed hardware ANN-AC equals to $700 \mathrm{CPU}$ cycles. And in an $8 \times 8$ mesh topology NoC, it averagely takes 200 $\mathrm{CPU}$ cycles to transfer packets in a round-trip journey. So the time duration between $a$ and $b$ is around 900 CPU cycles in our proposed system. The length of sampling window should be short enough to reflect the network status continuity between adjacent windows and also long enough to decrease the number of the packets caused by our mechanism and cover the control delay from $a$ to $b$ (900 CPU cycles), making Equation 5 viable. The length of sampling window is set as $20 \mathrm{k}$ CPU cycles.

$$
I R_{b c}=\frac{\text { control_rate } \times(c-a)-I R_{a b} \times(b-a)}{c-b}
$$

\section{EXPERIMENTS}

\subsection{Methodology}

We evaluate our technique with timing-detailed full-system simulator using PARSEC benchmarks [3]. For data validity, we only report results obtained from ROI execution in the experiments. We implement and integrate our design in GEM5 [4] with default configuration, in which the embedded network GARNET [2] and
NI are enhanced according to our technique illustrated before. A centralized ANN controller with a specific identifier, which is used for packets transfer, is realized in software and is connected to one of the central routers. The ANN related packets can be either transferred by a dedicated network or by the existing network. Since those packets only take a small percentage of the whole packets in NoC system (around $6 \%$ on average), to improve the resources utilization rate, we transfer the ANN related packets by the existing network. The key configurations of the simulation platform are shown in Table 2. Each PARSEC program runs on 64 cores with one core running one thread.

Table 2: Simulation platform configurations

\begin{tabular}{|c|c|l|}
\hline Item & Amount & Description \\
\hline \hline Processor & 64 cores & Alpha based 2GHz out-of-order cores. \\
\hline L1-Cache & 64 banks & $\begin{array}{l}\text { Private I/D caches, 32KB per-core, 4-way set } \\
\text { associative, 128B block size, 2-cycle latency. }\end{array}$ \\
\hline L2-Cache & 64 banks & $\begin{array}{l}\text { Chip-wide shared, 1MB bank size, 16-way set } \\
\text { associative, 128B block size, 6-cycle latency. }\end{array}$ \\
\hline Memory & 64 ranks & $\begin{array}{l}\text { 4GB DRAM, 8 controllers, 512MB rank, 16 } \\
\text { outstanding requests for each processor. }\end{array}$ \\
\hline NoC & 64 nodes & $\begin{array}{l}8 \times 8 \text { mesh network. Each node consists of 1 } \\
\text { router, network interface, core, L1 and L2 } \\
\text { caches. X-Y dimensional order routing. Router } \\
\text { is 2-stage pipelined, 4 VCs per virtual network } \\
\text { and 5 flits per VC. Directory based MOESI } \\
\text { protocol. A cache block consists of 1 packet. }\end{array}$ \\
\hline & 1 unit & $\begin{array}{l}\text { Connected to one of the central routers, 0.2 } \\
\text { GHz, 1088b packet information register file, } \\
120 b \text { input circular buffer, 12 ANN input nodes, } \\
10 \text { ANN hidden nodes, 10 ANN output nodes. }\end{array}$ \\
\hline
\end{tabular}

\subsection{Experimental results}

6.2.1 Application performance. In Figure 10, we compare the ROI finish time, throughput and network latency across 12 benchmarks in PARSEC. We normalize the non-optimized results as $100 \%$. By admission control, the ROI finish time averagely decreases to $82.2 \%$ (up to $76.2 \%$ ). Compared to the state-of-art fuzzy control mechanism proposed in [15] where the average decrease of ROI finish time is $11.2 \%$, our mechanism performs much better. By ANN-AC, the throughput in the ROI phase averagely increases by $20.9 \%$ and the network latency decreases to $76.0 \%$ (averagely $78 \%$ by fuzzy control).

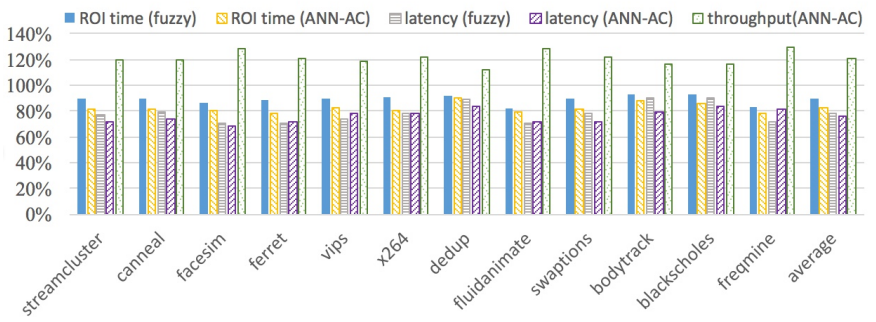

Figure 10: Comparison of system performance

6.2.2 Model accuracy. In our proposed model, we use a data preprocessing unit to decrease the inputs from 256 to 12 and a feature point extraction method for offline training. To illustrate the importance of the data preprocessing unit and feature point extraction method, in Figure 11, we present accuracy results produced by different ANN models (256-input and 12-input models illustrated in 
Section 4.2.2) and training data selection methods. For the 256-input ANN model, which is trained by the randomly selected data directly provided by profilers, the accuracy results are relatively low and begin to fluctuate when the number of training data is above 8000 . This is mainly because that there are too many weight parameters, requiring larger training set and that unnecessary information will make the weights deviate from the most appropriate results. For our proposed 12-input ANN model, if we choice training data randomly, the accuracy results will be lower than our proposed method. With the training data being larger, their difference becomes smaller. By our proposed model and training method, the predictive accuracy reaches the highest $90.2 \%$ with 12000 training data. So we can conclude that our proposed model and method can achieve higher predictive accuracy and require less training data.

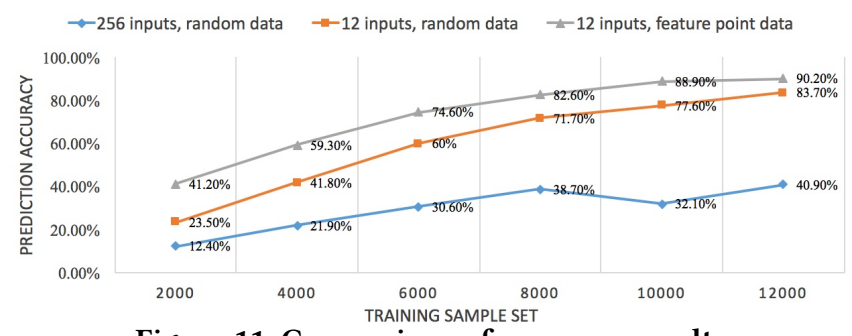

Figure 11: Comparison of accuracy results

6.2.3 Hardware evaluation. We implement our system in a 64-core chip with an $8 \times 8$ mesh network based on the CONNECT [17] open source router. We select the OpenRISC $120{ }^{1}$ open source $\mathrm{CPU}$ as the core model. The caches and NoC are adjusted according to Table 2. In our implementation, to decrease the area and power consumption, the frequency of $\mathrm{ANN}-\mathrm{AC}$ is set as $0.2 \mathrm{GHz}$ and its calculation latency is 70 cycles which equals to $700 \mathrm{CPU}$ cycles in our simulation configuration. We compare the hardware parameters of different units in a chip and the detailed hardware synthesis information is shown in Table 3. The ANN-AC consumes $47 \mathrm{k}$ equivalent NAND gates. The voltage is set to $1.1 \mathrm{~V}$ and the dynamic power consumption of the ANN-AC is only $1.80 \mathrm{~mW}$ which is much smaller than that consumed by one $\mathrm{CPU}$ or one router.

Table 3: Hardware synthesis results

\begin{tabular}{|c|c|c|c|}
\hline & Core & Router & ANN-AC \\
\hline Technology & \multicolumn{2}{|c|}{$40 \mathrm{~nm}$ low power, typical case } \\
\hline Number & 64 & 64 & 1 \\
\hline Frequency & $2 \mathrm{GHz}$ & $2 \mathrm{GHz}$ & $0.2 \mathrm{GHz}$ \\
\hline Gate count & $153 \mathrm{k}$ & $22 \mathrm{k}$ & $47 \mathrm{k}$ \\
\hline Dynamic power & $623.46 \mathrm{~mW}$ & $92.57 \mathrm{~mW}$ & $1.80 \mathrm{~mW}$ \\
\hline
\end{tabular}

\section{CONCLUSION}

We propose a centralized ANN-AC which can predict a proper flow regulation level. In the ANN-AC, a data preprocessing unit is used to prepare input data for the ANN predictor and a control rate generator to generate regulation rates. The experimental results show that the ANN-AC system can improve the ROI finish time averagely by $17.8 \%$ (maximum 23.8\%) when running the PARSEC benchmarks in full system simulations. Besides, the ANN-AC exhibits high prediction accuracy with less training data. Furthermore, we demonstrate the feasibility of the system implementation in hardware.

\section{REFERENCES}

[1] Ahmed Abdallah, Wayne Wolf, and Graham Hellestrand. 2008. Statistical characterization of execution time through simulation. In Proceedings of the 4th International Workshop on Intelligent Solutions in Embedded Systems (WISES).

[2] Niket Agarwal, Tushar Krishna, Li-Shiuan Peh, and Niraj K Jha. 2009. GARNET: A detailed on-chip network model inside a full-system simulator. In Proceedings of the International Symposium on Performance Analysis of Systems and Software (ISPASS).

[3] Christian Bienia, Sanjeev Kumar, Jaswinder Pal Singh, and Kai Li. 2008. The PARSEC benchmark suite: Characterization and architectural implications. In Proceedings of the 17th international conference on Parallel architectures and compilation techniques (PACT).

[4] Nathan Binkert, Bradford Beckmann, Gabriel Black, Steven K Reinhardt, Ali Saidi, Arkaprava Basu, Joel Hestness, Derek R Hower, Tushar Krishna, Somayeh Sardashti, et al. 2011. The gem5 simulator. ACM Transctions on SIGARCH Computer Architecture News 39, 2 (2011), 1-7.

[5] Ramazan Bitirgen, Engin Ipek, and Jose F Martinez. 2008. Coordinated management of multiple interacting resources in chip multiprocessors: A machine learning approach. In Proceedings of the 41st International Symposium on Microarchitecture (MICRO).

[6] Dominic DiTomaso, Ashif Sikder, Avinash Kodi, and Ahmed Louri. 2017. Machine learning enabled power-aware network-on-chip design. In Proceedings of the 21st Conference on Design, Automation \& Test in Europe (DATE).

[7] Simon Haykin. 1994. Neural networks: a comprehensive foundation. Prentice Hall PTR.

[8] Yoshi Shih-Chieh Huang, Kaven Chun-Kai Chou, Chung-Ta King, and Shau-Yin Tseng. 2010. Ntpt: On the end-to-end traffic prediction in the on-chip networks. In Proceedings of the 47th Design Automation Conference (DAC).

[9] Engin Ipek, Onur Mutlu, José F Martínez, and Rich Caruana. 2008. Self-optimizing memory controllers: A reinforcement learning approach. In ACM Transactions on SIGARCH Computer Architecture News, Vol. 36. 39-50.

[10] Fahimeh Jafari, Zhonghai Lu, Axel Jantsch, and Mohammad Hossein Yaghmaee. 2010. Buffer optimization in network-on-chip through flow regulation. IEEE Transactions on Computer-Aided Design of Integrated Circuits and Systems 29, 12 (2010), 1973-1986.

[11] Da-Cheng Juan, Siddharth Garg, Jinpyo Park, and Diana Marculescu. 2013. Learning the optimal operating point for many-core systems with extended range voltage/frequency scaling. In Proceedings of the 9th International Conference on Hardware/Software Codesign and System Synthesis.

[12] Elena Kakoulli, Vassos Soteriou, and Theocharis Theocharides. 2012. Intelligent hotspot prediction for network-on-chip-based multicore systems. IEEE Transactions on Computer-Aided Design of Integrated Circuits and Systems 31, 3 (2012), 418-431.

[13] Stefanos Kaxiras and Cliff Young. 2000. Coherence communication prediction in shared-memory multiprocessors. In Proceedings of the 6th International Symposium on High-Performance Computer Architecture (HPCA).

[14] Zhonghai Lu, Dimitris Brachos, and Axel Jantsch. 2009. A flow regulator for on-chip communication. In Proceedings of the 22nd Internetional SOC Conference.

[15] Zhonghai Lu and Yuan Yao. 2017. Dynamic Traffic Regulation in NoC-Based Systems. IEEE Transactions on Very Large Scale Integration (VLSI) Systems 25, 2 (2017), 556-569.

[16] Warren S McCulloch and Walter Pitts. 1943. A logical calculus of the ideas immanent in nervous activity. The bulletin of mathematical biophysics 5, 4 (1943), 115-133.

[17] Michael K Papamichael and James C Hoe. 2012. CONNECT: re-examining conventional wisdom for designing nocs in the context of FPGAs. In Proceedings of the ACM/SIGDA international symposium on Field Programmable Gate Arrays. ACM, 37-46.

[18] Liesbeth Steffens, Manvi Agarwal, and Pieter van der Wolf. 2008. Real-time analysis for memory access in media processing SoCs: A practical approach. In Proceedings of the 20th Euromicro Conference on Real-Time Systems (ECRTS). 255-265.

[19] Gordon Steven, Rubén Anguera, Colin Egan, Fleur Steven, and Lucian Vintan. 2001. Dynamic branch prediction using neural networks. In Proceedings of the 4th Euromicro Symposium on Digital Systems Design (Euro-DSD).

[20] Gervin Thomas, Ben Juurlink, and Dietmar Tutsch. 2011. Traffic prediction for NoCs using fuzzy logic. In Proceedings of the 2nd International Workshop on New Frontiers in High-performane and Hardware-aware Computing (HipHaC).

[21] MT Tommiska. 2003. Efficient digital implementation of the sigmoid function for reprogrammable logic. IEE Proceedings-Computers and Digital Techniques 150, 6 (2003), 403-411.

[22] Linda Wilson. 2013. International technology roadmap for semiconductors. IEEE Transactions on Semiconductor Industry Association (2013).

[23] Jae-Yeon Won, Xi Chen, Paul Gratz, Jiang Hu, and Vassos Soteriou. 2014. Up by their bootstraps: Online learning in artificial neural networks for CMP uncore power management. In Proceedings of the 20th International Symposium on High Performance Computer Architecture (HPCA) 